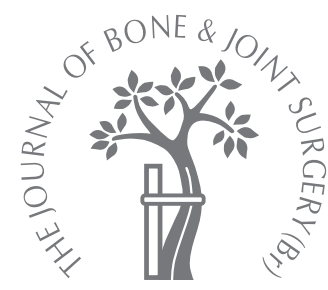

- CASE REPORT

\title{
Irreducible posterior subtalar dislocation with incarceration of a fracture of the anterior process of the calcaneum
}

\author{
H. Budd, \\ J. Wimhurst, \\ B. Davis, \\ R. Hutchinson
}

From Norfolk and

Norwich University

Hospital NHS Trust, UK

\footnotetext{
H. Budd, MBChB(Hons), MRCS(Eng), Specialist Registrar

Ipswich Hospital NHS Trust, Heath Road, Ipswich, Suffolk IP4 5PD, UK.

J. Wimhurst, FRCS, Consultant Orthopaedic Surgeon

B. Davis, FRCS, Consultant Orthopaedic Surgeon

R. Hutchinson, MD, FRCS, Consultant Orthopaedic Surgeon

Norfolk and Norwich University Hospital NHS Trust, Colney Lane, Norwich NR4 7UY, UK.

Correspondence should be sent to $\mathrm{Mr} \mathrm{H}$. Budd; e-mail: traumastudy@hotmail.com
}

(C)2010 British Editorial Society of Bone and Joint Surgery doi:10.1302/0301-620X.92B7. $23397 \$ 2.00$

$J$ Bone Joint Surg $[\mathrm{Br}]$ 2010;92-B:1025-7. Received 8 September 2009; Accepted after revision 5 February 2010

\begin{abstract}
Subtalar dislocation is a significant injury characterised by late complications, including subtalar arthritis. We describe a rare case of irreducible posterior subtalar dislocation due to incarceration of a fracture of the anterior process of the calcaneum in the subtalar joint, and discuss appropriate management.
\end{abstract}

Subtalar dislocation is defined by the presence of simultaneous dislocation of the talocalcaneal and talonavicular joints in the absence of a fracture of the neck of the talus. ${ }^{1}$ It is also known as peritalar or subastragalar dislocation, and is potentially complicated by instability, stiffness, late arthritis of the subtalar joint, and avascular necrosis of both the talus and the navicular, emphasising the need for early anatomical reduction. ${ }^{2}$ Four categories of subtalar dislocation are described, of which medial dislocation is the most common ( $72 \%$ to $86 \%)$, followed by lateral dislocation ( $12 \%$ to $22 \%)$. A few case reports have described anterior $(1 \%)$ and posterior dislocation $(1 \% \text { to } 2.5 \%)^{1,3-7}$ (Table I). These patterns of injury can combine, depending on the position of the foot and the direction of the force exerted at the time of injury, often making classification difficult. Posterior subtalar dislocation results from excessive plantar flexion of the foot, whereas medial and lateral dislocation have a rotational component and result from forced foot supination and pronation, respectively. We describe a case of irreducible posterior subtalar dislocation with incarceration of a fracture of the anterior process of the calcaneum in the subtalar joint.

\section{Case report}

A 26-year-old male motorcyclist was admitted with an irreducible closed subtalar dislocation of his right foot following a road traffic accident. The neurovascular status of the foot was not compromised and he had no other injuries. Anteroposterior and lateral plain radiographs demonstrated a posterior subtalar dislocation with slight lateral displacement and rotation of the foot (Fig. 1). Following failure of attempts at reduction in the emergency department, the patient was taken directly to the operating theatre for a further attempt at closed reduction under general anaesthetic in order to safeguard the medial soft tissue, where marked tension was observed in the skin due to prominence of the dislocated head of the talus.

The subtalar joint was manipulated, obtaining a completely stable reduction of the talonavicular joint, but the talocalcaneal joint appeared reduced but unstable. Fluoroscopic imaging in theatre demonstrated no incongruency of the talocalcaneal joint and the fracture of the anterior process of the calcaneum could not be appreciated. Once reduced, the talonavicular joint was maintained in a satisfactory position with percutaneous Kirschner wires passed from distal to proximal, thereby reducing the soft-tissue tension. A backslab was applied and a CT scan undertaken to elicit the underlying cause of the residual talocalcaneal instability. This showed separation of the subtalar joint by the fractured anterior process of the calcaneum, which measured $13 \mathrm{~mm} \times 26 \mathrm{~mm} \times$ $10 \mathrm{~mm}$. It had rotated through $180^{\circ}$ and displaced into the subtalar joint (Fig. 2).

The patient was returned to theatre 48 hours after the injury for open reduction of the talocalcaneal joint through a lateral sinus tarsi approach. The fragment of the anterior process of the calcaneum was retrieved from the subtalar joint and reduced before being secured with two headless compression screws. The small fragment of the anterior process to which the extensor digitorum brevis attached was brought back down and held using a bone suture anchor. The talonavicular, talocalcaneal and calcaneocuboid joints were all congruous, with the hindfoot stable to stress testing.

After surgery the patient was placed in a plaster-of-Paris backslab for ten days, which was then converted into a non-weight-bear- 
Table I. Classification of subtalar dislocation ${ }^{4}$

\begin{tabular}{lll}
\hline Type of dislocation & $\begin{array}{l}\text { Direction of foot displacement } \\
\text { in relation to talus }\end{array}$ & Total subtalar dislocations $\mathbf{( \% )} \mathbf{~}^{\mathbf{2 - 7}}$ \\
\hline Medial & Supination & 72 to 85 \\
Lateral & Pronation & 15 to 22 \\
Anterior & Dorsiflexion & 1 \\
Posterior & Plantar flexion & 1 to 2.5 \\
\hline
\end{tabular}

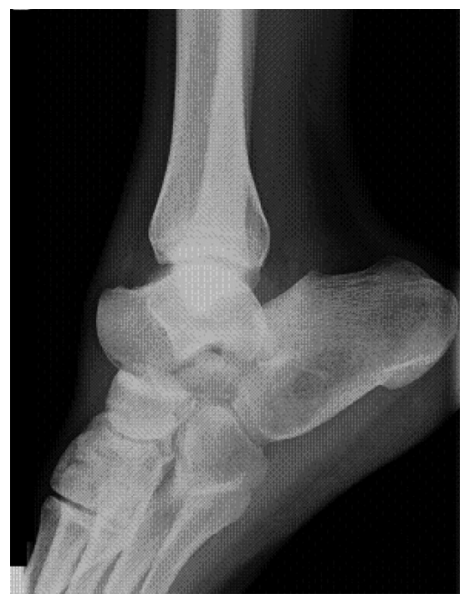

Fig. 1a

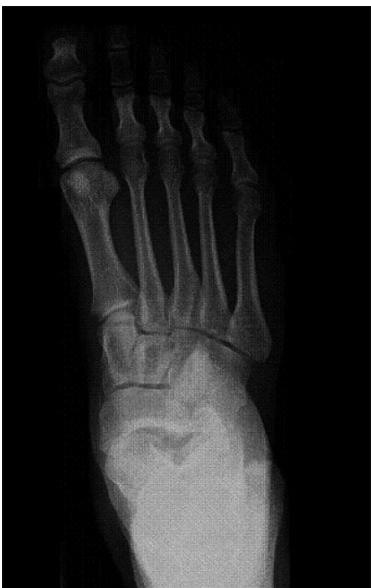

Fig. 1b

Radiographs of a) lateral view showing posterior subtalar dislocation with the head of the talus perched on the posterior aspect of the navicular, and b) anteroposterior view showing no medial or lateral displacement of the foot.

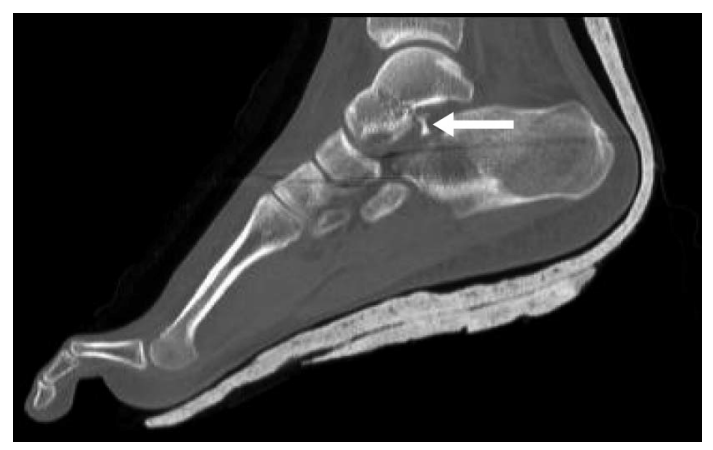

Fig. 2

CT scan showing subluxation of the subtalar joint and the incarcerated fracture (arrow) of the anterior calcaneal process.

ing cast for a further four weeks. By six weeks the wound was well healed and radiographs showed union of the fracture of the anterior process, with the presence of a Hawkin's sign demonstrating subchondral atrophy and excluding avascular necrosis. ${ }^{8}$ At 15 months the patient could walk bearing full weight, but with mild pain on inclines and hard ground. Subtalar movement was minimal and there was generalised osteopenia on plain

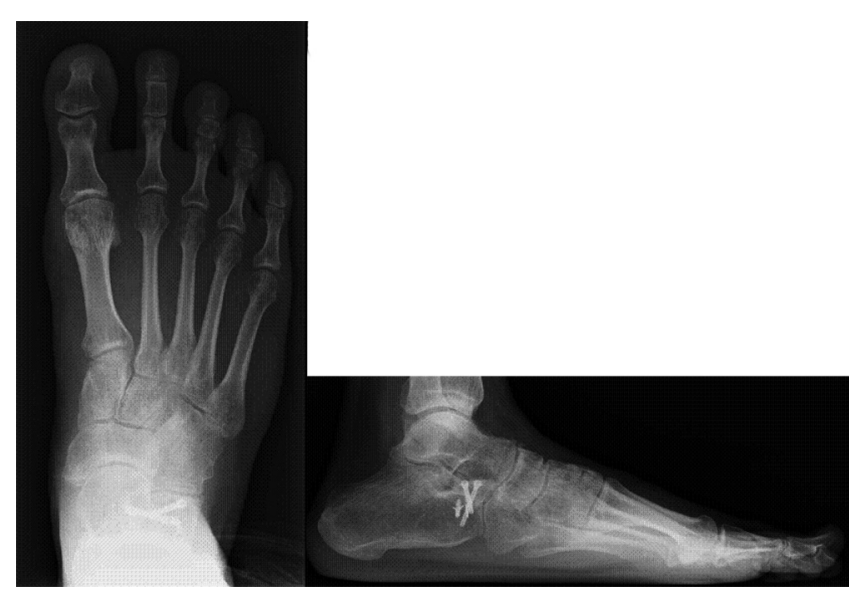

Fig. 3

Anteroposterior and lateral radiographs taken at 15 months' follow-up. The headless compression screws and suture anchor are seen. No early evidence of talonavicular osteoarthrosis can be seen.

radiographs, but no radiological evidence of early talonavicular osteoarthritis (Fig. 3).

\section{Discussion}

A posterior subtalar dislocation was defined radiologically by Inokuchi et $\mathrm{al}^{3}$ on a lateral view, where the head of the talus is seen perched on the posterior navicular with the posterior talus resting in the posterior subtalar facet of the calcaneum in the absence of any significant displacement or rotation of the foot. Posterior dislocation, albeit rare, is well described following injuries with excessive plantar flexion, but there is no previous description of an irreducible posterior subtalar dislocation due to incarceration of a fracture of the anterior process of the calcaneum. ${ }^{3,9,10}$

A fracture of the anterior process of the calcaneum is an uncommon injury which is often missed at initial presentation. ${ }^{11}$ One previous paper ${ }^{10}$ has described fracture with proximal dislocation into the subtalar joint in association with an Essex-Lopresti type B posterior facet joint depression injury of the calcaneum, but with preservation of the talonavicular joint. Ricci, Bellabarba and Sanders ${ }^{12}$ have described several cases of talonavicular dislocation associated with a severe crush fracture of the anterior process of the calcaneum, but these were attributed to plantar flexion of the head of the talus and dorsal dislocation of the 
talonavicular joint. Many obstacles to closed reduction of subtalar dislocation are described, including entrapment of the tendon of tibialis posterior around the neck and head of a fracture of the talus in a lateral dislocation, and obstruction by the tendons of extensor digitorum brevis and common extensors in medial dislocations. ${ }^{6}$ However, these are more typical of rotational dislocation and often respond to carefully directed manoeuvres in closed reduction. ${ }^{1,6}$ In the case we describe here, anatomical reduction was blocked by an incarcerated fragment of the anterior process of the calcaneum, which was only clearly demonstrated by CT and required open reduction and internal fixation.

Several mechanisms for fracturing the anterior process of the calcaneum have been postulated. It can be sheared off following forced abduction of the forefoot combined with strong hindfoot dorsiflexion. ${ }^{11}$ It can also be avulsed by traction on the bifurcate ligament, attaching it to the navicular and cuboid bones, during forced plantar flexion and supination of the foot, which is a plausible mechanism in the case we describe. The anterior process has also been described as acting as a fulcrum during lateral dislocation as the anterolateral corner of the talus is pressed against it.

This case highlights both the need for prompt reduction of soft-tissue tension by manipulation and bony stabilisation in complex foot injuries, and the necessity for further imaging following closed reduction to confirm joint congruency and anatomical reduction. Subtle joint subluxation and associated occult fractures may not be apparent on initial plain radiographs or during fluoroscopy after apparent reduction, but must always be considered. Following the initial reduction of a subtalar dislocation more detailed imaging, such as a thin cut CT, should be undertaken to ensure that an anatomical reduction has been achieved.

No benefits in any form have been received or will be received from a commercial party related directly or indirectly to the subject of this article.

\section{References}

1. De Lee J, Curtis R. Subtalar dislocation of the foot. J Bone Joint Surg [Am] 1982;64A:433-7.

2. Smith H. Subastragalar dislocation: a report of seven cases. J Bone Joint Surg 1937;19:373-80.

3. Inokuchi S, Hashimoto T, Usami N. Posterior subtalar dislocation. J Trauma 1997:42:310-13.

4. Saltzman C, Marsh JL. Hindfoot dislocations: when are they not benign? J Am Acad Orthop Surg 1997:5:192-8.

5. Broca P. Memoire sur les luxations sous-astragaliennes. Mem Soc Chir (Paris) 1853;3:566-656 (in French).

6. Leitner B. Obstacles to reduction in subtalar dislocations. J Bone Joint Surg [Am] 1954;36-A:299-306.

7. Zimmer TJ, Johnson KA. Subtalar dislocations. Clin Orthop 1989;238:190-4.

8. Hawkins LG. Fractures of the neck of the talus. J Bone Joint Surg [Am] 1970;52A:991-1002.

9. Krishnan KM, Sinha AK. True posterior dislocation of subtalar joint: a case report. J Foot Ankle Surg 2003;42:363-5.

10. Marcinko DE, Field N. Fractured anterior calcaneal process. J Foot Surg 1988;27:43-6.

11. Trnka HJ, ZettI R, Ritschl P. Fracture of the anterior superior process of the calcaneus: an often misdiagnosed fracture. Arch Orthop Trauma Surg 1998;117:300-2.

12. Ricci WM, Bellabarba C, Sanders R. Transcalcaneal talonavicular dislocation. J Bone Joint Surg [Am] 2002;84-A:557-61. 